\title{
Sargassum Polycystum C.Agardh Mediated Synthesis of Gold Nanoparticles Assessing its Characteristics and its Activity against Water Borne Pathogens
}

\author{
Sivaraj $\mathbf{R}^{1 *}$, Priya SVR ${ }^{2}$, Rajiv $\mathbf{P}^{2}$ and Rajendran $\mathbf{V}^{2}$ \\ ${ }^{1}$ Department of Biotechnology, School of Life Sciences, Karpagam University, Coimbator, India \\ ${ }^{2}$ Department of Chemistry, Government Arts College, Udumalpet, Tamilnadu, India
}

\begin{abstract}
This paper reports on the eco-friendly synthesis of gold nanoparticles (AuNPs) using the seaweed Sargassum polycystum C. Agardh extract. Biological synthesis for nanoparticle using plants is gaining considerable interest among researchers as an eco-friendly alternative to conventional physical and chemical methods, as this approach eliminates the use of toxic chemicals. Synthesized AuNPs was monitored by UV-Vis spectroscopy and was found to be complete within $30 \mathrm{~min}$. Confirmation of elemental gold was carried out by elemental mapping using different physical techniques such as Fourier transform infrared (FT-IR) spectroscopy, X-ray diffraction (XRD) and energy dispersive X-ray spectroscopy (EDX), Scanning electron microscopy (SEM). The bio reduced AuNPs exhibited remarkably good anti-bacterial activity against pathogens specifically Pseudomonas aeruginosa $(20 \mathrm{~mm})$ which is more susceptible. The elaborate experimental evidences support that the seaweed Sargassum polycystum C. Agardh extract can provide an environmentally benign rapid route for synthesis of AuNPs that can be applied for various purposes.
\end{abstract}

Keywords: X-ray diffraction; Optical properties; Nano structures

\section{Introduction}

Synthesis of gold nanoparticles (AuNPs) has gained immense significance during the last few years due to their catalytic, optical, and electrical properties [1]. Nanoparticles exhibit atom-like behaviours due to high surface energy resulting from high and large specific surface area, high fraction of surface atoms and wide gap between valence and conduction band when divided to near atomic size. The synthesis of AuNPs using an eco-friendly method is important to address the growing concerns on the overall toxicity of nanoparticles for medical and biotechnological applications. AuNPs have been considered important due to their unique and tunable surface plasmon resonance (SPR) property and their applications in biomedical science including drug delivery, tissue/tumour imaging, photo thermal therapy, immune chromatography, and identification of pathogens in clinical specimens [2]. Most of the available chemical processes for synthesis of gold nanoparticles (AuNPs) involve toxic chemicals that get adsorbed on the surface, leading to adverse effects in medical applications. Presently there is a growing need to develop environmentally benign process for rapid synthesis of nanoparticles [3]. The choice of an environmentally compatible solvent system, an eco-friendly reducing agent, and nonhazardous capping agents for the stabilization of the nanoparticles are three main criteria for a totally "green" nanoparticle synthesis. Concerning the biological application of nanoparticles it has been emphasized that methods of synthesis through biological system via, micro-organisms include bacteria, yeasts, fungi and diatoms synthesizing inorganic materials either intra or extracellularly would make the nanoparticles more biocompatible [4]. The uses of marine algae include traditional cosmetics, as antipyretic and antiseptic compounds, vermifuges and as treatments for sunstroke, coughs, haemorrhoids, stomach ailments, nose-bleeds, goitre and urinary diseases [5]. Sargassum polycystum belongs to Phaeophyta species. It is reported to be the most abundant brown algae and chief source of alginic acid. It has been suggested that environmental factors such as temperature, salinity, rainfall and $\mathrm{p}^{\mathrm{H}}$ influences the seasonal growth in Sargassum species. During recent years Sargassum from coastal water have been subjected to repeated annual [6]. Herein, we report the biogenic synthesis of AuNPs using aqueous extract of S.polycystum for reduction of $\mathrm{Au}$ ions. We demonstrated its antibacterial activity against various water borne pathogens.

\section{Materials and Methods}

\section{Collection of seaweed and extract preparation}

Sargassum polycystum was collected from the Gulf of Mannar, Rameshwaram $\left(9.2800^{\circ} \mathrm{N}, 79.3000^{\circ} \mathrm{E}\right)$, Tamil Nadu, India. The seaweed sample was washed in seawater and then fresh water to remove the epiphytes and other contamination. The sample was authenticated by Botanical Survey of India (BSI), Coimbatore (BSI/SRC/5/23/201415/Tech.169). The dry leaves were ground into fine powder in electric blender (Bajaj Model GX 11, Mumbai, India). $5 \mathrm{~g}$ of this powder was suspended in $100 \mathrm{~mL}$ of distilled water in a $300 \mathrm{~mL}$ Erlenmeyer flask followed by boiling for $15 \mathrm{~min}$. The extract obtained was filtered through Whatmann filter paper No.1 (50 mm; Sigma, Bangalore, India). The filtrate was collected and stored at $4^{\circ} \mathrm{C}$ which was used throughout all the experiments. The reaction was carried out at room temperature [7]. The reagents were of analytical grade obtained either from Merck (Mumbai, India) and Sisco Research Laboratories (Mumbai, India).

\section{Synthesis of AuNPS}

The reaction mixture was initially light brown and there was no

*Corresponding author: Sivaraj R, Department of Biotechnology, School of Life Sciences, Karpagam University, Coimbatore, India, Tel: 91-422-6471113; E-mail: rajeshwarishivaraj@gmail.com

Received February 17, 2015; Accepted March 16, 2015; Published March 28 2015

Citation: Sivaraj R, Priya SVR, Rajiv P, Rajendran V (2015) Sargassum Polycystum C.Agardh Mediated Synthesis of Gold Nanoparticles Assessing its Characteristics and its Activity against Water Borne Pathogens. J Nanomed Nanotechnol 6: 280. doi:10.4172/2157-7439.1000280

Copyright: ( 2015 Sivaraj R, et al. This is an open-access article distributed under the terms of the Creative Commons Attribution License, which permits unrestricted use, distribution, and reproduction in any medium, provided the original author and source are credited. 
Citation: Sivaraj R, Priya SVR, Rajiv P, Rajendran V (2015) Sargassum Polycystum C.Agardh Mediated Synthesis of Gold Nanoparticles Assessing its Characteristics and its Activity against Water Borne Pathogens. J Nanomed Nanotechnol 6: 280. doi:10.4172/2157-7439.1000280

turbidity formation during the course of time. Synthesis of AuNPs was carried out at the concentration of $50 \%$ against $1 \mathrm{mM} \mathrm{HAuCl}_{4}$ in a total volume of $100 \mathrm{ml}$ made up with double distilled water. Reduction of $\mathrm{Au}^{3+}$ ions was initiated by addition of $5 \mathrm{~mL}$ of extract to $95 \mathrm{~mL}$ of $10^{-3}$ $\mathrm{M}$ aqueous chloro auric acid solution in a $500 \mathrm{~mL}$ Erlenmeyer flask. The resulting mixtures were placed on a magnetic stirrer at the rate of $150 \mathrm{rpm}$ for $25 \mathrm{~min}$ and initial colour change was observed from light brown to ruby red colour.

\section{Characterization of AuNPs}

The color change in reaction mixture (metal ion solution+ alga extract) was recorded through visual observation. The bioreduction of gold ions in aqueous solution was monitored by periodic sampling subsequently measuring UV-Vis spectra of the solution at 500$600 \mathrm{~nm}$ using a $3-5 \mathrm{~mm}$ quartz cuvette. UV-Vis spectra of these aliquots were monitored as a function of time of the reaction. All the measurements were carried out at room temperature. All UV-visible (UV-vis) spectroscopic measurements of the synthesized AuNPs were carried out on a Cary 100 BIO UV-vis spectrophotometer (Varian, Palo Alto, CA, USA). AuNPs synthesized after $20 \mathrm{~min}$ of reaction between $1 \mathrm{~mm}$ chloroauric acid solution and extract were centrifuged at 10,000 rpm for $15 \mathrm{~min}$ at room temperature, following which the pellet was re-dispersed in sterile distilled water to remove any uncoordinated biological molecules. The chemical composition of the gold nanoparticles was characterized by FT-IR (Bruker Tensor 27). The phase formation of bio-reduced AuNPs was studied with the help of XRD. Bio reduced gold chloride solution was air dried. XRD patterns were recorded by a SEIFERT X-ray diffractometer with $\mathrm{Cu} \mathrm{K}$ a radiation. The samples were scanned in the $2 \theta$ range of $10^{\circ} \mathrm{C}-70^{\circ} \mathrm{C}$. Energy-dispersive X-ray (EDX) analysis was done using LEO $1430 \mathrm{VP}$, Carl Zeiss AG, Oberkochen, Germany to confirm the presence of gold in the particles, as well as to detect any other components, if present, performed on a SEM instrument(JEOL JSM 7500F Field Emission Scanning Electron Microscope).

\section{Antibacterial activity}

Antimicrobial activity of the crude extracts was determined by the agar well diffusion method [8]. All test organisms were inoculated in Mueller Hinton broth. Isolates were seeded on Mueller Hinton agar plates by using sterilized cotton swabs. Agar surface was bored by using sterilized gel borer to make wells ( $7 \mathrm{~mm}$ diameter). $100 \mu \mathrm{l}$ of the test extract and $100 \mu \mathrm{l}$ of sterilized distilled water (negative control) were poured in to separate wells. The standard antibiotic tetracycline was placed on the agar surface as positive control. Plates were incubated at $37^{\circ} \mathrm{C}$ for 48 hours. Triplicate plates were maintained for each organism. The plates were examined for evidence of zones of inhibition which appear as a clear area around the wells. The diameter of such zones was measured using a meter ruler and the mean value for each organism was recorded and expressed in millimeter [9].

\section{Results and Discussion}

Reduction of $\mathrm{Au}^{3+}$ to AuNPs could be followed by colour change from yellow to ruby red and further by UV-Vis spectroscopy. The peak observed at $540 \mathrm{~nm}$ confirmed the synthesis of AuNPs as it is in agreement with the previous reports [10]. Various techniques used for characterization produced characteristic results for the AuNPs. Sargassum polycystum aqueous extract to $1 \mathrm{mM} \mathrm{HAuCl} \mathrm{H}_{4}$ solution lead to the appearance of ruby red colour indicating the formation of gold nanoparticles. These colours arise due to the excitation of Surface Plasmon Vibrations in the metal nanoparticles. The absorption maxima for the biosynthesized nanoparticles were noted in the visible range of 500-560 nm. The gold Surface Plasmon Resonance (SPR) band occurred at $532 \mathrm{~nm}$ and steadily increased in intensity as the different concentration of reaction medium without any shift in the peak (wave-length). Reduction of $\mathrm{Au}^{3+}$ to AuNPs by GGFE could be followed by colour change from yellow to ruby red and further by UVVis spectroscopy. The peak observed at $532 \mathrm{~nm}$ confirmed the synthesis of AuNPs as it is in agreement with the previous reports. The presence of carboxylic, amine, phosphate and hydroxyl functional groups was confirmed by FT-IR measurements (Figure 1). FT-IR is an important parameter to check the functional groups present and stabilization of nanoparticles. The range of Au nanoparticles falls between 800-1400 which confirms the presence of $\mathrm{Au}$ nanoparticles. These peaks present in the synthesis of nanoparticle may also indicate that seaweed extract may play a role in stabilizing AuNPs by adsorbing on the surface of bio reduced AuNPs. Analysis via Energy Dispersive X-ray (EDX) spectrometers confirmed the presence of elemental gold signals of the $\mathrm{Au}-\mathrm{NPs}$ (Figure 2). The phase formation of the synthesized AuNPs was analysed employing X-ray diffraction which confirmed that the bio reduced metal nanoparticles are of elemental gold existence of peaks (111), (200), (220) and (311) matched with the standard Joint Committee for Powder Diffraction Set (JCPDS). In similar works, the presence of intense peaks of nanoparticles (111), (200) and (211) appeared which are indexed as crystalline silver centered cubic phase, the XRD pattern thus clearly shows that the silver nanoparticles formed by the reduction of $\mathrm{Ag}^{2+}$ ions by S. polycystum extract are crystalline [11] (Figure 3). The XRD patterns of green-synthesized Turbinaria conoides nanoparticle revealed that AuNPs corresponded to the crystalline gold fcc phase. The diffraction peaks obtained at $2 \theta=38.36^{\circ}(111), 44.13^{\circ}(200), 64.78^{\circ}$ (220), and $77.98^{\circ}(311)$ are identical with those reported for standard gold metal (Joint Committee on Powder Diffraction Standards-JCPDS, USA) [12]. Similarly, the XRD patterns of green-synthesized Gnidia glauca flower extract revealed existance of peaks (111), (200), (220) and (311) matched with the standard Joint Committee for Powder Diffraction Set (JCPDS) data-04784. This confirmed face centered cubic structured AuNPs formation. Peak broadening indicated restricted particle size. Enlarged pattern of (111) peak is shown in the inset of XRD plot [13]. Seaweed extract alone does not bring any effects in the shape and size of the particles, the phytochemicals present in the extracts may have the role of synthesizing the nanoparticles. Using the Scherrer's formula the size of the nanoparticle has been calculated. $\mathrm{d}=0.9 \lambda / \beta \cos \theta$ Here 0.9 is the shape factor, generally taken for a cubic system, $\lambda$ is the X-ray wavelength, typically $1.54 \AA$, $\beta$ is the full width at half the maximum intensity (FWHM) in radians, and $\theta$ is the Bragg angle. Using the above formula the crystallite size calculated is $\sim 15$ $\mathrm{nm}$. The surface of gold nanoparticles and the elements present in the extract were analysed by EDX (Figure 2). The AuNPs were mono dispersed as spherical shaped. The average size of the particles is between 30-60 nm (Figure 4). A few agglomerated particles were also observed due to the spectral shift. Antibacterial activity of S. polycystum extract, gold nanoparticles and their combination were tested against E.coli and P.aeruginosa organism and the activity is shown in Figure 5. Antibacterial activity of $S$. polycystum extract, silver nitrate and their combination were tested against human pathogens also.

The crude extract of $S$. polycystum and silver nanoparticles showed cytotoxicity against MCF 7 breast cancer cell line in dose dependent manner. The IC50 for seaweed extract at $300 \mathrm{mg} \mathrm{mL}^{-1}$ for $72 \mathrm{~h}$ was higher than other concentration and the IC50 for Silver nanoparticles at $135 \mathrm{mg} \mathrm{mL}^{-1}$ for $72 \mathrm{~h}$ was higher than other concentration $[14,15]$. 
Citation: Sivaraj R, Priya SVR, Rajiv P, Rajendran V (2015) Sargassum Polycystum C.Agardh Mediated Synthesis of Gold Nanoparticles Assessing its Characteristics and its Activity against Water Borne Pathogens. J Nanomed Nanotechnol 6: 280. doi:10.4172/2157-7439.1000280

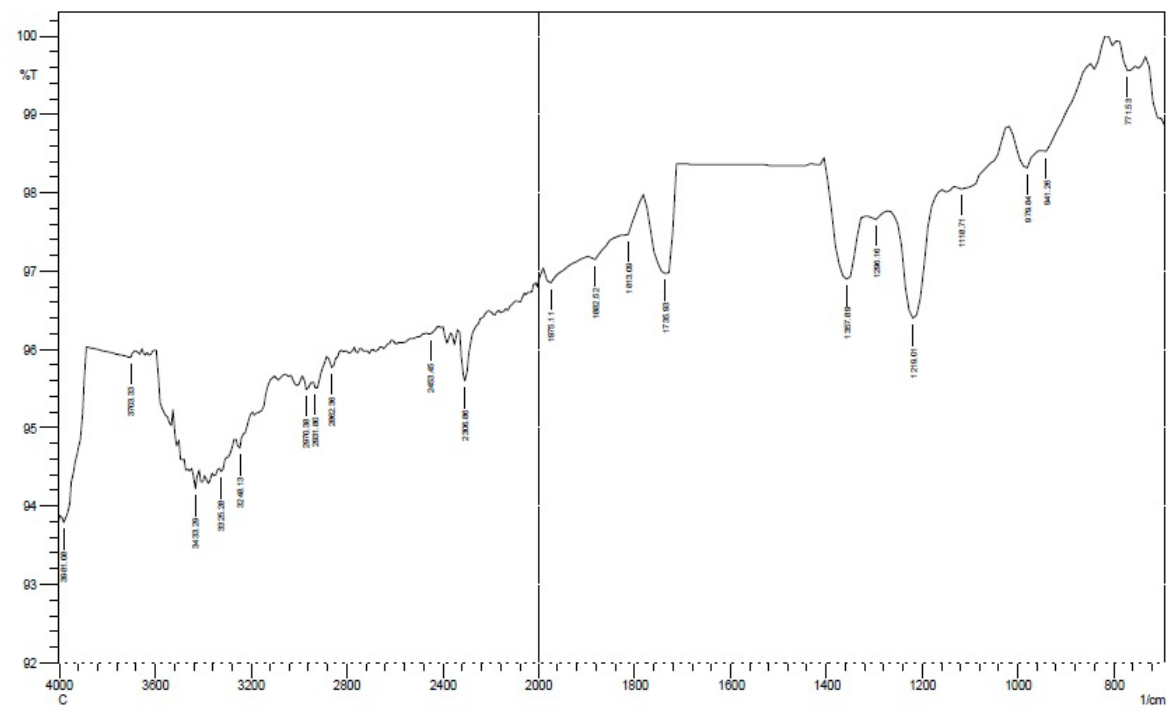

Figure 1: FT-IR spectra of gold nanoparticles.

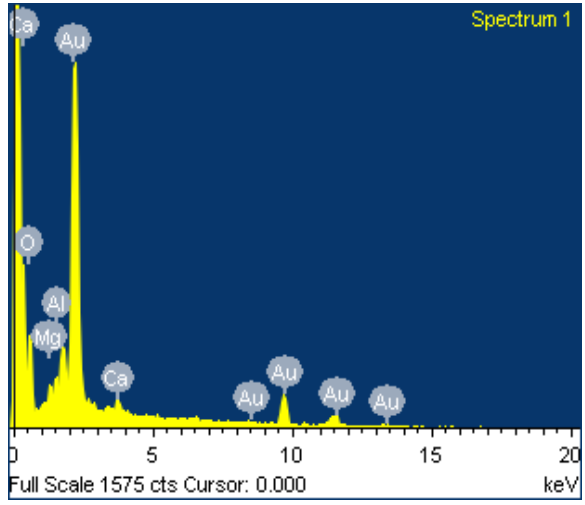

Figure 2: EDX analysis of Gold Nanoparticles.

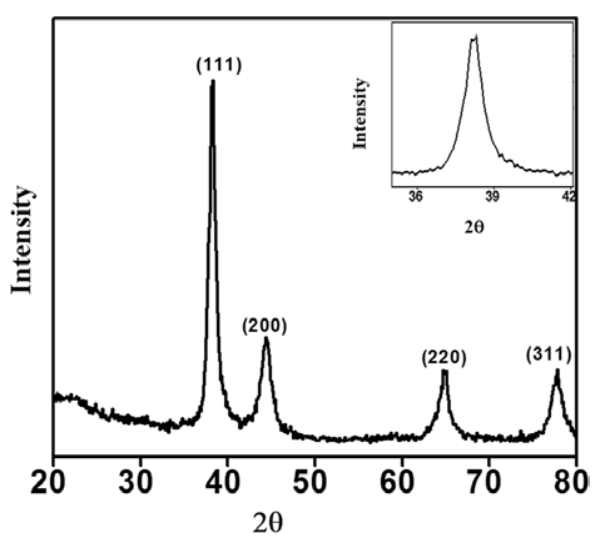

Figure 3: XRD image of gold nanoparticles.

\section{Conclusion}

Green synthesis of spherical shaped AuNPs has been achieved using extract of $S$. polycystum thus bringing into light yet another use

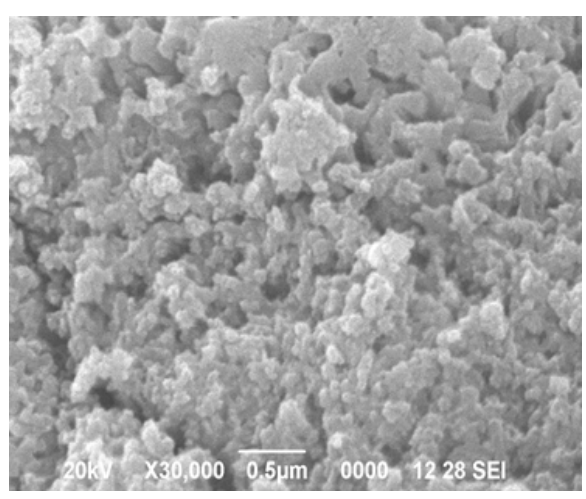

Figure 4: SEM image of gold nanoparticles.

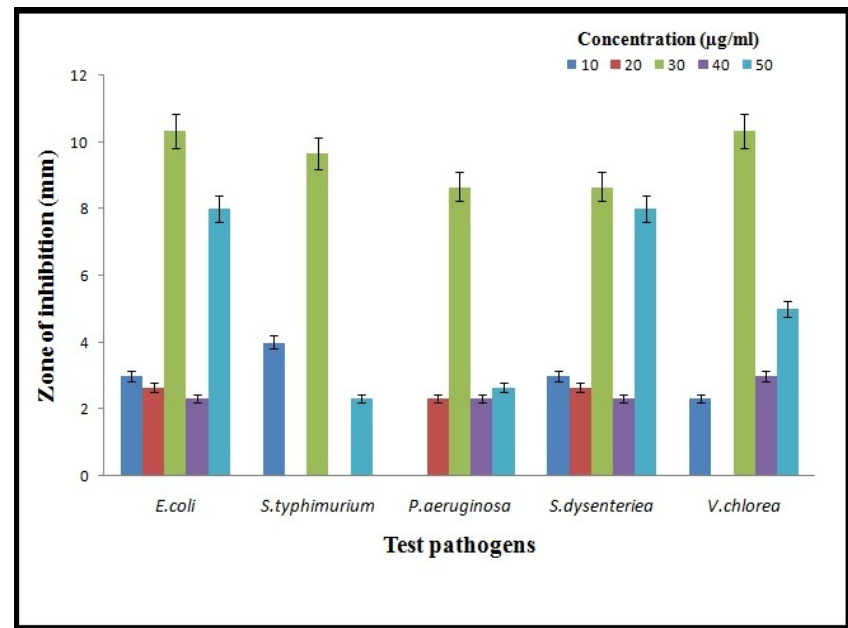

Figure 5: Values are expressed as mean \pm standard deviation of thethree replicates. 
Citation: Sivaraj R, Priya SVR, Rajiv P, Rajendran V (2015) Sargassum Polycystum C.Agardh Mediated Synthesis of Gold Nanoparticles Assessing its Characteristics and its Activity against Water Borne Pathogens. J Nanomed Nanotechnol 6: 280. doi:10.4172/2157-7439.1000280

of the plant, besides its usual utilities. The method stands out primarily due to the fact that it is eco-friendly and shuts down the demerits of conventional physical and chemical methods. These particles are anticipated to have an extensive application in various biomedical applications. Thus this rapid, eco-friendly and economical route can be used to synthesize AuNPs with wide biotechnological and chemical applications.

\section{Acknowledgement}

The support and facility provided by the Management of Karpagam University, is acknowledged.

\section{References}

1. Kasthuri J, Kathiravan K, Rajendiran NJ (2009) Phyllanthin-assisted biosynthesis of silver and gold nanoparticles: a novel biological approach. $J$ Nanopart 11: 1075-1085.

2. Gleiter $\mathrm{H}(2000)$ Nanostructured materials: basic concepts and microstructure. Acta Materialia 48: 1-29.

3. Ghosh S, Patil S, Ahire M, Kitture R, Jabgunde A, et al. (2011) Synthesis of gold nanoanisotrops using Dioscorea bulbifera tuber extract. J Nanomater 7 483-496.

4. Govindaraju K, Basha SK, Kumar V, Kumar G, Singaravelu G, et al. (2008) Silver, gold and bimetallic nanoparticles production using single cell protein Spirulina platensis (Geitler). J Mater Sci 43: 5115-5122.

5. Ahmad A, Mukherjee P, Senapati S, Mandal D, Khan Ml, et al. (2003) Extracellular biosynthesis of silver nanoparticles using the fungus Fusarium oxysporum. Colloids and Surf B: Biointerfaces 28: 313-318.
6. Rao AS, Rao MU (2002) Seasonal growth pattern Sargassum polycystum c.agardh (Phaeophyta, Fucales) occurring at Visakhapatnam, east coast of India 31: 26-32.

7. Thangaraju N (2012) Synthesis of silver nanoparticles and the antibacterial and anticancer activities of the crude extract of sargassum polycystum c. Agardh. Nano Biomed and Eng 4: 89-94.

8. Tagg TR, Dajani AS, Wannamaker LW (1976) Bacteriocin of Gram positive bacteria. Bacteriological Reviews 40: 722-756.

9. Kalaiselvi M, Subbaiya R, Masilamani Selvam (2013) Synthesis and characterization of silver nanoparticles from leaf extract of Parthenium hysterophorus and its anti-bacterial and antioxidant activity. Int J Curr Microbio App Sci 2: 220-227.

10. Ghosh S, Patil S, Ahire M, Kitture R, Jabgunde A, et al. (2011) Synthesis of gold nano-anisotrops using Dioscorea bulbifera tuber extract. J Nanomaterials: $1-8$

11. Kandhasamy M, Arunachalam KD (2008) Evaluation of invitro antibacterial property of seaweeds of Southeast coast of India. African J. Biotech 7: 19581961.

12. Rajeshkumar S, Malarkodi S, Gnanajobitha G, Paulkumar K, Vanaja M, et al. (2013) Seaweed-mediated synthesis of gold nanoparticles using Turbinaria conoides and its characterization. J Nanostru Chem 3: 44.

13. Ghosh S, Patil S, Ahire M, Kitture R, Gurav DD, et al. (2012) Gnidia glauca flower extract mediated synthesis of gold nanoparticles and evaluation of its chemocatalytic potential. J Nanobiotech 10: 17.

14. Li WR, Xie XB, Shi QS, Duan SS, Ouyang YS, et al. (2011) Antibacterial effect of silver nanoparticles on Staphylococcus aureus. Biometals 24: 135-141.

15. Malarkodi E, Manoharan A (2013) Study on antibacterial activity of Parthenium hysterophorus L. J Chem Pharm 5: 137-139. 